\title{
The optimism surrounding stereotactic body radiation therapy and immunomodulation
}

DOI:

$10.21037 / \mathrm{cco} .2017 .05 .01$

Link to publication record in Manchester Research Explorer

\section{Citation for published version (APA):}

Tharmalingam, H., \& Hoskin, P. J. (2017). The optimism surrounding stereotactic body radiation therapy and immunomodulation. Chinese clinical oncology, 6(Suppl 2), S9. https://doi.org/10.21037/cco.2017.05.01

\section{Published in:}

Chinese clinical oncology

\section{Citing this paper}

Please note that where the full-text provided on Manchester Research Explorer is the Author Accepted Manuscript or Proof version this may differ from the final Published version. If citing, it is advised that you check and use the publisher's definitive version.

\section{General rights}

Copyright and moral rights for the publications made accessible in the Research Explorer are retained by the authors and/or other copyright owners and it is a condition of accessing publications that users recognise and abide by the legal requirements associated with these rights.

\section{Takedown policy}

If you believe that this document breaches copyright please refer to the University of Manchester's Takedown Procedures [http://man.ac.uk/04Y6Bo] or contact uml.scholarlycommunications@manchester.ac.uk providing relevant details, so we can investigate your claim.

\section{OPEN ACCESS}




\title{
The optimism surrounding stereotactic body radiation therapy and immunomodulation
}

\author{
Hannah Tharmalingam, Peter J. Hoskin \\ Mount Vernon Cancer Centre, Northwood, UK \\ Contributions: (I) Conception and design: None; (II) Administrative support: None; (III) Provision of study materials or patients: None; \\ (IV) Collection and assembly of data: None; (V) Data analysis and interpretation: None; (VI) Manuscript writing: All authors; (VII) Final approval of \\ manuscript: All authors. \\ Correspondence to: Prof Peter J. Hoskin. Mount Vernon Cancer Centre, Rickmansworth Road, Northwood, Middlesex HA6 2RN, UK. \\ Email: peterhoskin@nhs.net.
}

\begin{abstract}
In recent years, rapidly evolving radiation techniques have enabled the precise delivery of very high doses of radiation to local targets with stereotactic ablative body radiotherapy (SABR). In addition to its direct cytotoxicity, radiation and in particular SABR has powerful immunomodulatory effects resulting in immunogenic cell death and potentiation of the anti-tumour immune response. However, due to the immunosuppressive nature of non-irradiated sites of metastases, radiotherapy alone is seldom sufficient to induce the systemic response required for distant tumour rejection. Immune checkpoint inhibitors are a novel class of immunomodulatory agents shown to have robust efficacy against a number of malignancies. These drugs can augment the effects of radiotherapy by helping overcome tumour-induced immunosuppression at local and distant sites. Similarly, radiation may complement immunotherapy by priming tumours in preparation for the adaptive immune response, thereby leading to more prolonged clinical effects. This synergistic relationship has been demonstrated in laboratory models and has been extended to a number of early phase clinical studies.
\end{abstract}

Keywords: Abscopal effect; immunotherapy; stereotactic body radiotherapy; tumour immunity

Submitted Mar 25, 2017. Accepted for publication Apr 19, 2017.

doi: $10.21037 /$ cco.2017.05.01

View this article at: http://dx.doi.org/10.21037/cco.2017.05.01

\section{Introduction}

Recent advances in our understanding of the biology of the anti-tumour immune response have rapidly accelerated progress in cancer immunotherapy. Immune-checkpoint inhibitors in particular have provided encouraging clinical responses across a number of different tumour sites. However, many of those patients who initially respond eventually develop resistant disease (1). One way to improve the effectiveness and durability of immunotherapy may be to combine it with radiotherapy.

Radiotherapy is a fundamental oncological treatment playing a crucial role in the definitive and adjuvant settings as well as in the palliation of tumour-related symptoms across a wide variety of malignancies. Although initially thought to be immunosuppressive, a significant body of evidence now exists to suggest that radiotherapy has potent immunomodulatory effects, enhancing tumour immunogenicity and augmenting anti-tumour immune responses both locally and systemically (2). Whilst conventionally delivered in small daily doses over multiple weeks, recent technological advancements have allowed for the precise local delivery of much larger doses of radiation with stereotactic ablative body radiotherapy (SABR) offering the potential for yet further radiation-induced tumour immunogenicity and immunogenic cell death.

In this review, we discuss the biological rationale behind combining stereotactic radiation and immunotherapy as well as the extensive pre-clinical and evolving clinical evidence in support of this approach. We then focus on 
current clinical trials and highlight the likely challenges that may lie ahead in this new field.

\section{The host immune system and tumorigenesis}

The unique ability of the adaptive human immune system to differentiate between self and non-self allows it to recognise the presence of cells undergoing malignant transformation. The immunosurveillance hypothesis predicts that a competent immune system can selectively eliminate cancer cells and protect against the development of solid malignancies (3). Tumours only become clinically apparent once they acquire the ability to evade immune destruction, thought predominantly to be due to the creation of an immunosuppressive local tumour microenvironment (4). Fundamental to this is the vasculature of the tumour which it commonly outgrows resulting in abnormal angiogenesis, hypoxia and ischaemia. The subsequent upregulation of hypoxia inducible factor-1 (HIF-1) stimulates the local production of the stromal-derived factor-1 (SDF-1) chemokine which in turn drives the recruitment of myeloidderived suppressor cells (MDSCs) and tumour-associated macrophages (TAMs) $(5,6)$. The immunosuppressive actions of these myeloid cells is multi-faceted. They secrete IL-10, an anti-inflammatory cytokine shown to activate regulatory $\mathrm{T}$ cells (Tregs) (7) and induce a tolerogenic phenotype amongst intratumoral dendritic cells (DCs) (8). This culminates in the presentation of tumour antigens to cytotoxic T lymphocytes (CTLs) without the required co-stimulatory molecules needed to activate a response, ultimately resulting in anergy of the CTLs $(9,10)$. Other secretory factors include TGF-beta and reactive oxygen and nitrogen species which directly blunt the effector function of CTLs (10), as well as arginase and nitric oxide that deplete $\mathrm{L}$-arginine, an essential metabolite for $\mathrm{T}$ cell function (10-12). Finally, MDSCs express the programmed death ligand-1 (PD-L1) (13) which engages with the programmed death-1 (PD-1) receptor on activated CTLs to induce their exhaustion (14). Blockade of this pathway has been shown to re-invigorate CTLs and restore their antitumour cytotoxic activity $(15,16)$ hence it has become a key target in current immunotherapy strategies.

\section{Immunomodulatory therapy}

The PD-1/PDL-1 axis is one of a number of 'immune checkpoint' pathways hijacked by tumours as a means of evading the host immune response. A number of tumour types over-express PD-L1 and its increased expression is thought to correlate with worse prognoses across various tumour sites (17). A second important immune checkpoint is the cytotoxic T-lymphocyte associated protein-4 (CTLA-4) which engages with CD80/CD86 on the surface of intratumoral DCs. Activation of CTLs following presentation of tumour antigens by DCs requires costimulation driven by the cellular ligation of CD80/CD86 to CD28 on CTLs. The high affinity of CTLA-4 to CD80/ CD86 acts as a competitive inhibitor to this interaction thereby suppressing the anti-tumour immune response (18). In recent years, monoclonal antibody $(\mathrm{mAb})$ therapies against both CTL4-A and PD-1 have been employed as immune checkpoint inhibitors with promising clinical responses across a range of tumour sites. The first was ipilimumab, an anti-CTL4-A mAb approved in 2011 having been shown to have a dramatic effect on the overall survival (OS) of patients with metastatic melanoma. The pivotal study by Hodi et al. demonstrated that patients treated with ipilimumab and a peptide vaccine had an OS of 10 months compared to only 6.4 months for those who received the vaccine alone (19). Further studies have demonstrated durable clinical responses of up to 10 years in some cases (20), a significant improvement in a disease with previously dismal long-term survival rates.

Nivolumab is a selective mAb targeting the PD-1 receptor and has also shown to be effective in numerous tumour sites. In a phase III study of BRAF wild-type patients with advanced melanoma, those receiving nivolumab had a 1 -year survival rate of $72.9 \%$ compared to $42.1 \%$ in those given dacarbazine chemotherapy (21). In metastatic renal cell carcinoma, nivolumab improved OS to 25 months compared to 19.6 months in those treated with everolimus (22), and in comparison with docetaxel as a second-line treatment for metastatic non-small cell lung cancer (NSCLC), superior OS rates and toxicity profiles with nivolumab were demonstrated across histologies in two randomised controlled phase 3 trials (Checkmate 017 and Checkmate 057) $(23,24)$. In the latter, improved OS, progression-free survival (PFS) and objective response rates were all seen to correlate with tumoral PD-L1 expression with nivolumab nearly doubling median overall survival in patients whose tumours expressed PD-L1 (24). Unselected 1 -year OS survival rates increased from $39 \%$ to $51 \%$ and the trial was terminated early having been adjudged to have met its primary end point of improved overall survival (24).

Similarly exciting results have been seen with pembrolizumab, another $\mathrm{mAb}$ against the $\mathrm{PD}-1$ receptor. 
When compared to ipilimumab in the treatment of advanced melanoma, pembrolizumab doubled the median progression-free survival and improved 1-year OS rates from $58.2 \%$ to $74.1 \%$ with a significantly better acute toxicity profile (25). In light of such positive clinical results, a number of newer agents targeting the PD-1/PD-L1 pathway are currently being investigated in early phase trials (26).

Although immunotherapies have produced impressive clinical data thus far, durable responses are only seen in a small minority of patients with most eventually developing resistant disease. It is proposed that long-term remission rates may be improved by combining immunomodulation with radiotherapy. Whilst immune checkpoint inhibition works by overcoming immune evasion mechanisms employed by the tumour, an initial priming of the immune system against specific tumour-associated antigens (TAAs) is still required in order to generate a reaction of sufficient scale and quality to adequately target the solid malignancy (1). New biological insights into the immunogenicity of radiation therapy suggest that it may serve as an ideal tool to prime the adaptive anti-tumour immune response in this way, thereby enhancing the efficacy of immunotherapies when the two modalities are used in synergy.

\section{Radiation therapy and the immune system}

Traditional radiobiological teaching has focussed on the direct cytocidal effects of radiotherapy arising from the generation of irreparable double-stranded DNA breaks resulting in mitotic catastrophe and cellular apoptosis (27). There is now a significant body of evidence that radiation therapy also has potent immunomodulatory effects, orchestrating a spectrum of cellular and molecular alterations culminating in the potentiation of the systemic anti-tumour immune response. Radiation-induced immunogenic cell death is thought to be mediated through the heightened release of tumour-associated antigens (TAAs) generated by apoptotic and necrotic cell debris (28), as well as pro-inflammatory 'danger' signals and damageassociated molecular patterns (DAMPs) such as heat-shock proteins (HSPs) (29), and high-mobility group protein B1 (HMGB1) (30). DAMPs and 'danger' molecules act via toll-like receptors (TLRs) to stimulate dendritic cells, facilitating the uptake of TAAs and their presentation on major histocompatibility complex class 1 (MHC-1) for the activation of tumour-specific cytotoxic $\mathrm{T}$ lymphocytes $(31,32)$. This process is further augmented by the radiation- induced translocation of calreticulin (CRT) to the cell surface membrane of tumour cells which has been shown to significantly increase tumour cell sensitivity to the cytolytic effects of CTLs (33). CRT also interacts via CD91 on DCs and macrophages to stimulate receptor-mediated phagocytosis of tumour antigens and their subsequent presentation on MHC-1 (34). Exposure to CRT has been shown to be a critical requirement for radiation-induced immunogenic cell death (35). Finally, radiation therapy has been shown to upregulate MHC-1 expression in both normal tissue and tumour cells (36), again facilitating the presentation of tumour-specific antigens to CTLs and stimulating their immune-mediated destruction.

In addition to its cellular effects, ionising radiation also has significant influence over the local tumour microenvironment. It has been shown to induce the expression of inflammatory chemokines, notably CXCL10 and CXCL16 which stimulate the recruitment of CD4 and CD8 T lymphocytes to the sites of the tumour $(37,38)$. Radiation also interacts with the tumour vasculature, upregulating adhesion molecules such as vascular cell adhesion protein 1 (VCAM-1) and intracellular adhesion protein 1 (ICAM-1) which are critical for local leukocyte transmigration (39). Taken together, these effects promote the attraction and infiltration of anti-tumour CTLs into the tumour microenvironment, repressing its immunosuppressive nature and enhancing the local immune-mediated destruction of the irradiated tumour.

As well as acting locally, it makes biological sense that a radiation-induced systemic immune response against common tumour epitopes shared by primary and metastatic lesions may also underlie the abscopal effect. First reported by Mole in 1953, the abscopal effect describes the phenomenon whereby targeted irradiation is seen to cause regression of distant metastases outside of the radiation field (40). However, abscopal responses are seldom seen in clinical practice in response to radiotherapy alone thought to be due to the immunosuppressive nature of the tumour microenvironment at distant, untreated sites (41). It logically follows therefore that repression of coinhibitory immune molecules such as CTLA-4 and PD-L1 might help overcome this, further supporting the rationale for combining radiation therapy with systemic immune checkpoint blockade.

\section{Preclinical evidence for combining radiation with immunotherapy}

The synergistic effect of immune therapies and radiotherapy 
on tumour control has consistently been demonstrated in pre-clinical studies. In the poorly immunogenic 4T1 mouse model of metastatic breast cancer, combining an anti-CTLA-4 mAb with radiotherapy delivered in one or two fractions of 12 Gy resulted in superior local and distant tumour regression compared to either modality alone with a significantly improved OS (42). The increased survival was seen to correlate with the inhibition of distant lung metastasis and was dependent on the presence of CD8positive $T$ cells (42). These results were consistent with those seen in a murine glioma model where the synergistic effects of CTLA-4 blockade and focal radiotherapy again prolonged OS (43). Triple therapy with the addition of an agonist antibody against $4-1 \mathrm{BB}$, a co-stimulatory signal activating CTLs resulted in yet further improvement, an effect seen to be predominantly CD4-positive, rather than CD8-positive T cell-mediated (43).

Preclinical data also supports the strategy of combining radiotherapy with $\mathrm{PD}-1$ inhibition. In a triple-negative breast cancer mouse model, tumours were cured when anti-PD-1 mAbs were combined with single or low-dose fractionated radiotherapy and CD8-positive $\mathrm{T}$ cells were seen to be critical to this curative response (44). Data from a mouse glioma model corroborated these results, with administration of anti-PD-1 therapy significantly enhancing the efficacy of stereotactic radiosurgery (SRS), increasing the local intra-tumoral infiltrate of cytotoxic $\mathrm{T}$ cells and improving overall survival compared to either treatment modality alone (45).

\section{Radiation dose and immunogenicity}

Conventional radiotherapy has traditionally been delivered in small-dose (1.8-3 Gy), multi-fraction regimes over a number of weeks. Recent technological advancements in 4-dimensional planning systems and image-guidance have resulted in the development of SABR, a technique which allows for the precise delivery of much higher doses (6-30 Gy) of radiation either as a single treatment or in a small number of fractions.

A number of in vitro studies across various tumour sites have demonstrated that higher radiation doses such as those typically employed in SABR induce significantly greater anti-tumour immune responses and thereby improve local tumour control compared to lower doses $(36,46,47)$. One study examining the cytolytic effects of the delivery of 0,10 and 20 Gy to human prostate, colon and lung carcinoma cells in vitro showed the higher dose to induce greater immune-mediated cell killing across all three cell lines (45). Irradiation of a B16 mouse melanoma model with single doses of 5, 7.5, 10 and 15 Gy also demonstrated a dosedependent improvement in local tumour control, although this was mildly offset at the highest dose by an increased representation of regulatory $\mathrm{T}$ cells (47). Mechanisms underlying this dose-dependent effect include a greater expression of stimulatory immune molecules and tumour antigens such as carcinoembryonic antigen (CEA) on the surface of malignant cells (46), up-regulation of MHC-1 with increased presentation of tumour epitopes to $\mathrm{T}$ cells (36) and an increased infiltration of tumour-specific CD8-positive $\mathrm{T}$ cells into the tumour microenvironment and the draining lymph nodes (47). All culminate in the enhanced immune recognition of tumours and their cytotoxic destruction. The improvements in local control and survival are lost when stereotactic radiotherapy is administered to mice depleted of CD8-positive T cells (48), providing further credence to the hypothesis that immunomodulatory effects are fundamental in mediating the therapeutic benefits of SABR.

\section{Radiation fractionation and immunogenicity}

In addition to radiation dose, fractionation is also thought to affect immunogenicity. In terms of local control, studies typically show larger, single-dose regimes to be superior or at least equivalent to those delivered in multiple lowerdose fractions (47-49). Comparison of a single 20 Gy with four 5 Gy fractions in a murine melanoma model showed the single dose to impede growth in $100 \%$ of tumours and induce complete regression in $35 \%$, whilst the fractionated regime, albeit a lower biological equivalent dose (BED), had no beneficial effect (48). This response was again seen to be CD8-positive T cell-dependent and correlated with the infiltration of tumour-specific CTLs both locally and systemically into the draining lymph nodes (48). These results were consistent with those of another study utilising a similar mouse model comparing a single 15 Gy treatment with five 3 Gy fractions (49). Additional adoptive transfer studies performed by this group demonstrate that an increased number of tumour-specific $T$ cells remain within tumours taken from mice irradiated with a single dose whilst in those taken from mice treated with the fractionated regimen, $\mathrm{T}$ cell numbers appear to diminish over time (49). It is possible to infer from this data that 
single-dose regimens may result in anti-tumour immune effects of greater quality and amplitude resulting in a prolonged memory response which may translate into more durable long-term clinical outcomes.

The aforementioned studies demonstrate the benefit of single-dose regimens solely in terms of local tumour control and only make a comparison with multiple, low-dose fractionations. Subsequent investigations examining the effects on distant metastases suggest that SABR-like highdose per fraction ( $\geq 6-8 \mathrm{~Gy}$ ) regimes result in enhanced systemic anti-tumour responses compared to single dose therapy. Much of the evidence for this arises from studies utilising the previously described 4T1 mouse breast cancer model where malignant cells spontaneously metastasize to the lungs (42). Mice were treated with either two $12 \mathrm{~Gy}$ fractions or a single 12 Gy dose to the primary tumour, concurrent with anti-CTLA-4 therapy. Both regimes were seen to significantly reduce the number of lung metastases and extend overall survival, but the survival advantage was significantly improved after two radiation fractions (42). Building on these results, the investigators then went on to test various dose-fractionation combinations to identify the most optimal in effecting a systemic anti-tumour response (50). Experiments were performed using two murine models, one with MC38 colon carcinoma cells and the other with TSA breast cancer cells. A primary and secondary tumour were implanted in both models and the primary lesion irradiated with either five 6 Gy fractions, three 8 Gy fractions or a single 20 Gy dose. Anti-CTLA4 was delivered post-irradiation and regression of the primary and secondary tumours were evaluated reflecting local and distant responses respectively. In terms of local control, all three regimes were equally as effective (50). However, in combination with immunotherapy, only the fractionated schedules were seen to elicit an abscopal response. Secondary tumour regression induced by the three 8 Gy regime was shown to be far superior to that of the five $6 \mathrm{~Gy}$ fractionation. The inhibition of the secondary tumour was proportional to its infiltration by interferon- $\gamma$ secreting CD8-positive $\mathrm{T}$ cells and a higher frequency of these cells was also reported in the spleens of the mice irradiated with the three 8 Gy schedule (50). This would suggest that this regime resulted in a superior disseminated immune response ultimately leading to an enhanced abscopal effect. Taken together, the results of these investigations suggest that high-dose, fractionated radiotherapy regimes analogous to those used clinically in SABR are the most systemically immunogenic and therefore may be the best for use in combination with immunotherapies.

\section{Clinical evidence for combining SABR with immunotherapy}

To date, a number of case reports demonstrating abscopal effects from combining immunotherapy with SABR have been published (51-55). The first pivotal case was described in a 33 year-old patient with metastatic melanoma who received SABR (delivered in three fractions of $9.5 \mathrm{~Gy}$ ) to a painful paraspinal mass, in conjunction with ipilimumab (51). Radiological evaluation revealed not only a significant response in the target lesion, but also considerable regression of disease outside the radiation field with complete resolution of metastatic splenic lesions and a substantial decrease in hilar lymphadenopathy. These responses were noted to be durable, with the disease remaining stable a further two years on. A similar case was reported on later that year where ipilimumab was administered either side of SABR (54 Gy in three fractions) to two metastatic liver lesions in a man with disseminated melanoma (52). A complete systemic response was observed, despite the patient having previously progressed on ipilimumab monotherapy. The following year saw the first case of the abscopal effect in a patient with metastatic non-small cell lung cancer when a 64 year-old man received stereotactic radiotherapy delivered in five 6 Gy fractions to a liver metastasis, in conjunction with ipilimumab immunotherapy (53). A complete response was again seen with total regression of the irradiated lesion as well as that of distant disease in the lungs and bone. Finally, in a patient with metastatic melanoma, ipilimumab combined with brain stereotactic radiosurgery also induced a complete systemic response in the skin and lymph nodes (54). Consistent with this last report are the results of a retrospective analysis of patients with advanced melanoma comparing combination treatment of ipilimumab with either SRS or whole-brain radiotherapy (WBRT) for brain metastases. For those receiving SRS, median overall survival was seen to be 19.9 months, compared to only 3.1 months for those receiving WBRT (55). These results appear to suggest superior efficacy of stereotactic regimens when used in combination with immunotherapy, although the possible selection of medically fitter patients with more favourable prognoses for treatment with SRS over WBRT should also be considered here. 


\section{Current clinical trials investigating the combination of SABR and immunotherapy}

Following the dramatic abscopal effects described in the discussed case reports, several clinical trials combining SABR with immunotherapy are now currently active. MD Anderson Cancer Centre have recently reported on a prospective phase I trial of patients with disseminated solid malignancies evaluating ipilimumab given either concurrently or before SABR to at least one primary or metastatic lesion in the lung, liver or adrenal gland. Preliminary results suggest that all therapeutic combinations tested were feasible and safe with distant abscopal effects also seen in 31 out of the 35 participants (56). Phase II of this trial is now recruiting to provide further efficacy data. The RADVAX trial led by researchers at the University of Pennsylvania is a dose-limiting toxicity study looking at SABR to a single metastatic lesion followed by ipilimumab in patients with advanced-stage melanoma (57). A similar phase 1 trial is currently enrolling at Johns Hopkins University assessing the safety of the combination of ipilimumab delivered either side of robotic arm SABR in melanoma patients with newly diagnosed spinal or brain metastasis (58). In Europe, the Dutch-led randomised phase II PEMBRO-RT study is prospectively investigating the delivery of pembrolizumab following SABR versus pembrolizumab alone in patients with metastatic nonsmall cell lung cancer (59), whilst the UK-based PERM study intends to evaluate the efficacy of the combination of SABR and pembrolizumab in metastatic melanoma (60). Numerous other comparable clinical trials are currently ongoing worldwide in this area, the results of which are expected to report over the next few years (Table 1).

\section{Unanswered questions and future perspectives}

Preliminary findings to date suggest there may be significant clinical benefit in combining SABR with immunotherapy, but there are a variety of issues that still need to be addressed as the field advances. Firstly, the optimal sequencing of the two treatment modalities. Some advocate prior administration of radiation in line with the biological hypothesis that SABR acts to 'prime' the immune system, generating sufficient TAAs to activate DCs and tumourspecific cytotoxic $\mathrm{T}$ lymphocytes before augmentation of the cytolytic response by immunotherapies (69). Moreover, due to its cytotoxic effects on lymphocytes, delivering radiotherapy after immune stimulation may in fact be detrimental rather than beneficial to the ongoing anti-tumour immune response (69). Contrariwise, administration of immunotherapy first would allow for the presence of an activating immune agent within the tumour microenvironment prior to radiotherapy. Prestimulation of DCs and CTLs locally may make them more readily available to respond to the surge in tumour-specific epitopes generated by SABR and consequently maximise its efficacy (69). It has been proposed that the mechanism of action of the selected immunotherapy agent might determine the most appropriate sequencing of modalities and this hypothesis is supported by a pre-clinical (70) study in a murine model of colorectal adenocarcinoma. Tumourbearing mice were treated with a single dose of $20 \mathrm{~Gy}$ combined with either an anti-CTL4-A antibody or an antiOX40 agonist antibody administered either before or after the dose of radiation. OX40 is a tumour necrosis factor (TNF) receptor expressed predominantly on activated cytotoxic $\mathrm{T}$ lymphocytes and transmits a powerful costimulatory signal once engaged. Anti-CTL4-A therapy was most effective when given prior to radiotherapy, noted in part to be due to a depletion of regulatory $\mathrm{T}$ cells within the local tumour microenvironment (70). Conversely, anti-OX40 agonist treatment proved most optimal when delivered one day post-radiotherapy during the window of increased tumour-associated antigen presentation and radiation-induced inflammation (70). Taken together, these data suggest that the most appropriate timing of the two treatment modalities may be dependent on the mechanism of action of the immunomodulatory agent used and further studies to improve our understanding in this area are clearly required.

Identification of the patient populations most likely to benefit from combination immuno-radiotherapy is another critical area that needs to be addressed. SABR is currently most commonly employed in the curative treatment of patients with stage 1 NSCLC and in the setting of oligometastatic disease. In the context of the former, monotherapy with SABR results in local tumour control of $\geq 95 \%$ (71) with relatively low regional lymph-node and distant recurrence rates of $5-10 \%$ and $15 \%$ respectively (72). It is hypothesised that the low incidence of distant spread following SABR may be a consequence of the elimination of micrometastases via the induction of a systemic anti-tumour immune response (69). It makes biological sense that augmentation of this response by the addition of activating immunotherapy may further reduce regional and distant relapses and theoretically lead to increased rates of cure. 


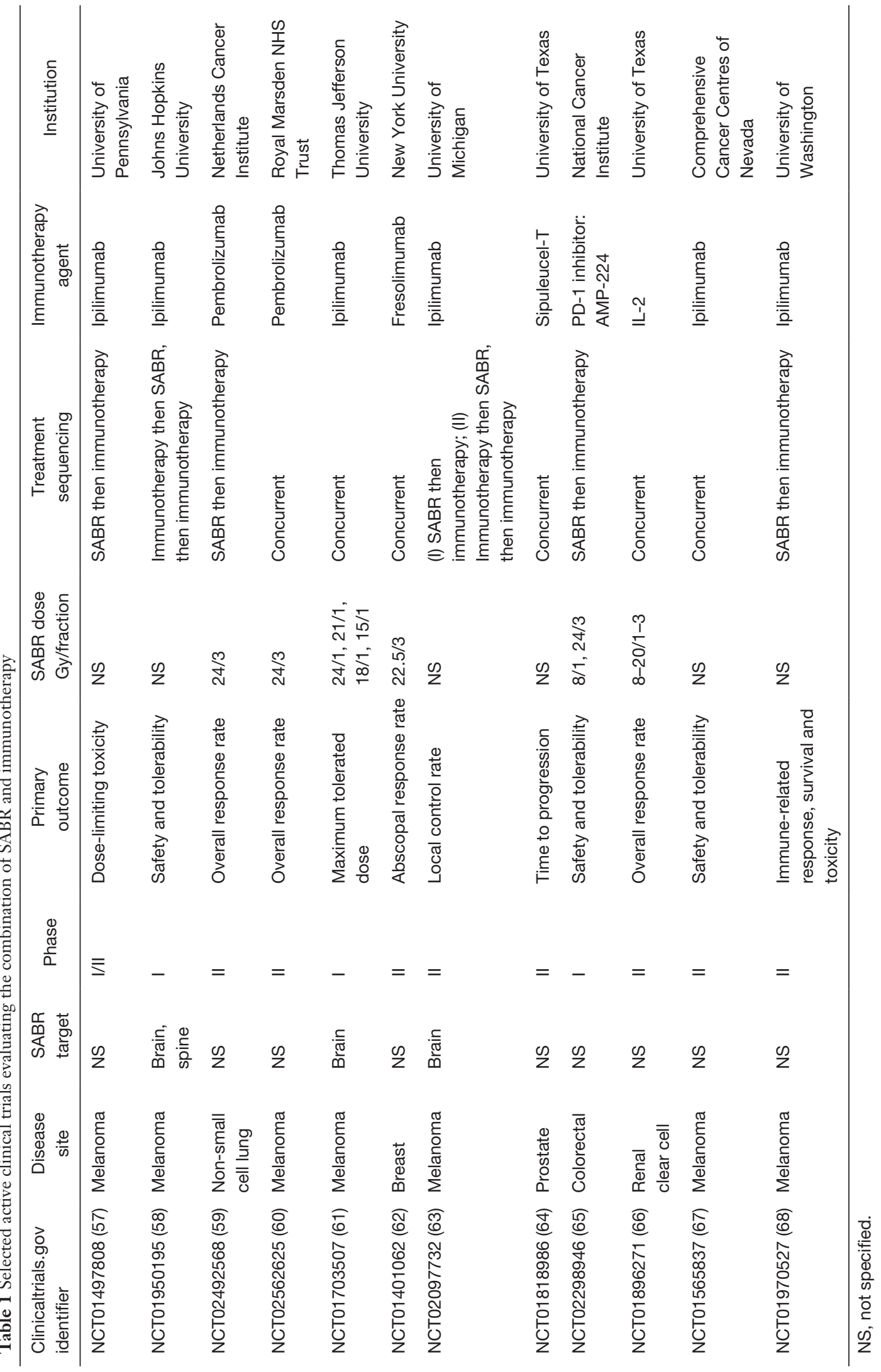


The relatively new paradigm of the oligometastatic state describes an intermediate stage in the disease history of the many solid tumours whereby only a small number of metastases develop initially, before the eventual acquisition of widespread dissemination potential and polymetastatic disease. SABR is being employed with increasing frequency as a radical local treatment for oligometastases with the aim of long-term disease control and potentially even cure. A number of non-randomised studies have consistently demonstrated excellent 1- to 2-year in-field control rates of up to $95 \%$ when treating oligometastases with SABR (73-75). However, there remains a significant risk of distant relapse with 2- to 5 -year disease-free survival rates approaching only $20 \%$ (76) suggesting a proportion of this patient cohort harbour subclinical metastases. Combining SABR with immunotherapy in this context may elicit the systemic immune response required to target and possibly eliminate the non-visible tumour burden thereby delaying disease progression, potentially improving overall survival rates.

Case reports describing the remarkable abscopal effects in patients with more extensive metastatic disease suggest that this cohort may also benefit from combination SABR and immunotherapy treatment. However, in one of the few phase III trials evaluating this approach in patients with metastatic prostate cancer, a survival benefit was only seen in a subgroup of men with favourable prognostic factors, most notably a smaller disease burden and the absence of visceral metastasis (77). It is possible that the systemic immune effects arising from combining radiation with immunotherapy may be more effective in the elimination of subclinical rather than bulky metastasis and perhaps our initial focus should be in those with more limited disease.

This review has focused on combining radiotherapy with immune-checkpoint inhibition, but there are a number of other immune strategies in various stages of development. These include cytotoxic gene therapy utilising oncolytic viruses (78), chimeric antigen receptor $\mathrm{T}$ cells $(79,80)$ and a variety of therapeutic cancer vaccines $(81,82)$. Sipuleucel $\mathrm{T}$ is an immune vaccine consisting of APCs activated $e x$-vivo by a recombinant fusion protein made up of prostatic antigens fused to an immune-cell activator. In the treatment of castrate-resistant metastatic prostate cancer, monotherapy with sipuleucel $\mathrm{T}$ has been shown to improve overall survival in three double-blinded, randomised controlled trials (83-85). Potentially augmenting this response further by combining such cancer vaccines with radiation is an exciting prospect for the future.
Finally, an important point highlighted by the trials with sipuleucel $\mathrm{T}$ is the difficulty in monitoring the clinical response to immunotherapy. The IMPACT trial saw a significant improvement in overall survival despite no objective response being observed on serial computer tomography and bone scanning (85). The initial response to immunotherapy can be notoriously difficult to interpret, with $\mathrm{T}$ cell infiltration and inflammation resulting in the enlargement or development of new lesions commonly misinterpreted as disease progression. Taking this into consideration, immune-related response criteria as an alternative to conventional RECIST measures in evaluating anti-tumour responses to immunotherapy have been proposed (Table 2) (86), although these criteria may fail to fully characterize all relevant patterns of clinical activity. It is accepted that the identification of generic immune biomarkers may prove difficult given the highly individualised nature of tumour mutations and MHC haplotypes. Nevertheless, future efforts should be directed towards identifying such immunological readouts to supplement the more traditional measures used to assess response which may not provide a complete and accurate evaluation of immunotherapeutic agents.

\section{Conclusions}

The ability of radiation therapy to elicit a systemic antitumour immune response is not a novel concept, nor is the proposal to combine radiotherapy with immunotherapy, the benefits of which have long been demonstrated in laboratory studies. Recent advances in our understanding of T-cell immune biology and regulatory processes have led to the development of systemic immune-checkpoint inhibitors that are providing consistent responses in the clinic. Preclinical studies suggest that SABR-like dosing regimens may be the most immunogenic and therefore the most optimal in augmenting the anti-tumour immune response when given in combination with immune-checkpoint inhibitors. Almost all clinical case reports demonstrating abscopal effects resulting from combination treatment used doses consistent with SABR. There are currently well over 60 published and ongoing clinical trials investigating this synergistic therapeutic approach with many more anticipated over the coming years. A number of challenges remain, particularly with regards to the most optimal sequencing of treatment, appropriate patient selection and accurate monitoring of disease response. The results of current research efforts will undoubtedly provide vital further information to drive 
Table 2 Comparison between the RECIST 1.1 and the irRC criteria in the evaluation of tumour response (adapted from Wolchok et al. 2009)

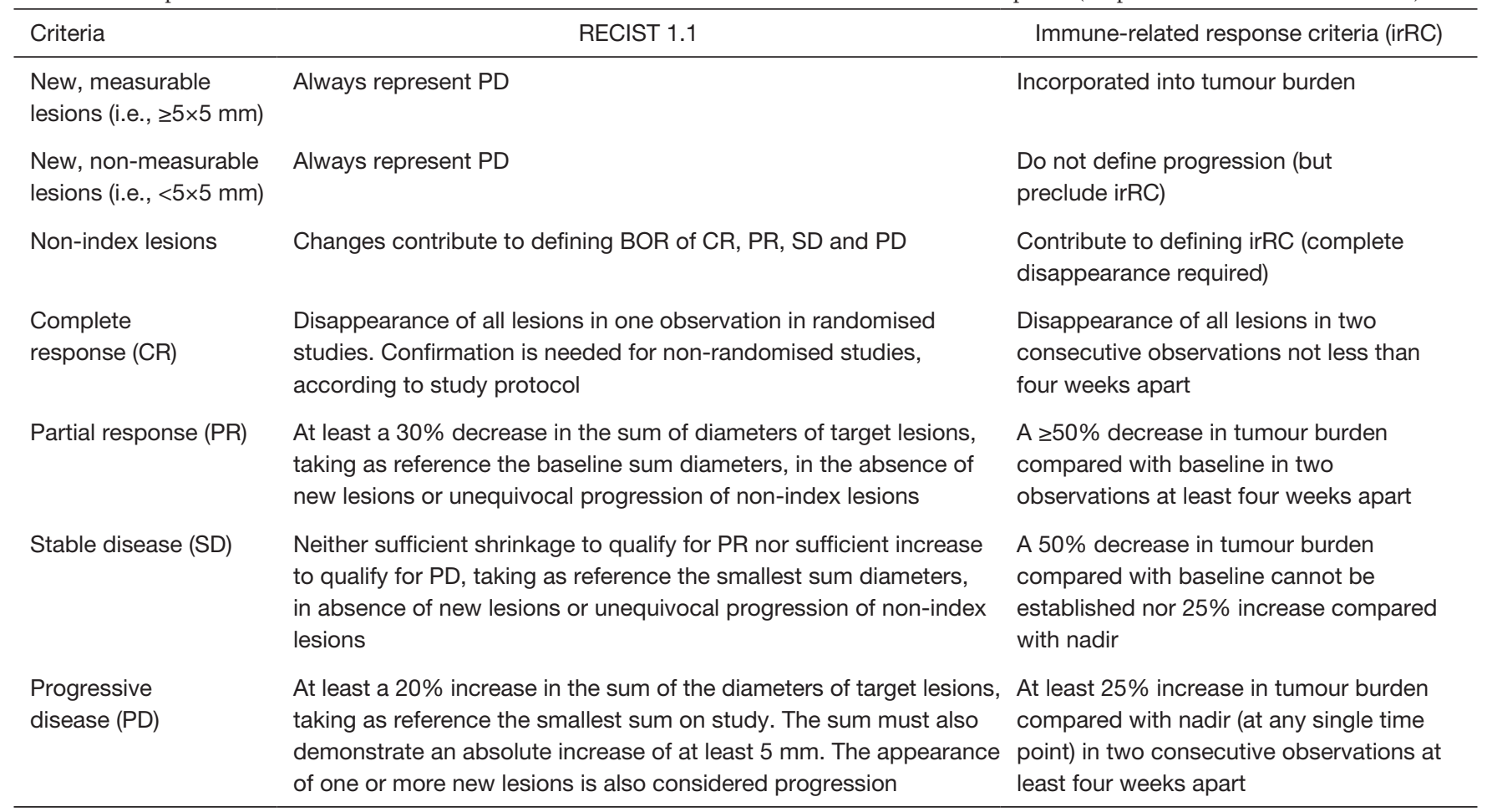

RECIST, response evaluation criteria in solid tumours; irRC, immune-related response criteria; PD, progressive disease; BOR, best overall response; $\mathrm{CR}$, complete response; $\mathrm{PR}$, partial response; $\mathrm{SD}$, stable disease.

forward our clinical practice within this exciting new field of combination immuno-radiotherapy.

\section{Acknowledgements}

None.

\section{Footnote}

Conflicts of Interest: The authors have no conflicts of interest to declare.

\section{References}

1. Brooks ED, Schoenhals JE, Tang C, et al. Stereotactic ablative radiation therapy combined with immunotherapy for solid tumors. Cancer J 2016;22:257-66.

2. Salama AK, Postow MA, Salama JK. Irradiation and immunotherapy: From concept to the clinic. Cancer 2016;122:1659-71.

3. Finn OJ. Immuno-oncology: understanding the function and dysfunction of the immune system in cancer. Ann
Oncol 2012;23 Suppl 8:viii6-viii9.

4. Chow MT, Moller A, Smyth MJ. Inflammation and immune surveillance in cancer. Semin Cancer Biol 2012;22:23-32.

5. Ceradini DJ, Kulkarni AR, Callaghan MJ, et al. Progenitor cell trafficking is regulated by hypoxic gradients through HIF-1 induction of SDF-1. Nat Med 2004;10:858-64.

6. Sánchez-Martín L, Estecha A, Samaniego R, et al. The chemokine CXCL12 regulates monocyte-macrophage differentiation and RUNX3 expression. Blood 2011;117:88-97.

7. Seo N, Hayakawa S, Takigawa M, et al. Interleukin-10 expressed at early tumour sites induces subsequent generation of CD4+ T-regulatory cells and systemic collapse of antitumour immunity. Immunology 2001;103:449-57.

8. Steinbrink K, Jonuleit H, Muller G, et al. Interleukin-10treated human dendritic cells induce a melanoma-antigenspecific anergy in CD8+ T cells resulting in a failure to lyse tumor cells. Blood 1999;93:1634-42.

9. Groux H, Bigler M, de Vries JE, et al. Interleukin-10 induces a long-term antigen-specific anergic state in 
human CD4+ T cells. J Exp Med 1996;184:19-29.

10. Gabrilovich DI, Ostrand-Rosenberg S, Bronte V. Coordinated regulation of myeloid cells by tumours. N Engl J Med 2012;12:253-68.

11. Bronte V, Serafini P, Mazzoni A, et al. L-arginine metabolism in myeloid cells controls T-lymphocyte functions. Trends Immunol 200324:302-6.

12. Zea AH, Rodriguez PC, Atkins MB, et al. Arginaseproducing myeloid suppressor cells in renal cell carcinoma patients: a mechanism of tumor evasion. Cancer Res 2005;65:3044-8.

13. Noman MZ, Desantis G, Janji B, et al. PD-L1 is a novel direct target of HIF-1 and its blockade under hypoxia enhanced MDSC-mediated T cell activation. J Exp Med 2014;211:781-90.

14. Chikuma S, Terawaki S, Hayashi T, et al. PD-1-mediated suppression of IL-2 production induces CD8+ $\mathrm{T}$ cell anergy in vivo. J Immunol 2009;182:6682-9.

15. Parekh VV, Lalani S, Kim S, et al. PD-1/PD-L blockade prevents anergy induction and enhances the anti-tumour activities of glycolipid-activated invariant NKT cells. J Immunol 2009;182:2816-26.

16. Chang WS, Kim JY, Kim YJ, et al. Cutting edge: Programmed death-1/programmed death ligand 1 interaction regulates the induction and maintenance of invariant NKT cell anergy. J Immunol 2008;181:6707-10.

17. Wu P, Wu D, Li L, et al. PD-L1 and survival in solid tumours: a meta-analysis. PLoS One 2015;10:e0131403.

18. Peggs KS, Quezada SA, Allison JP. Cancer immunotherapy: co-stimulatory agonists and co-inhibitory antagonists. Clin Exp Immunol 2009;157:9-19.

19. Hodi FS, O’Day SJ, McDermott DF, et al. Improved survival with ipilimumab in patients with metastatic melanoma. N Eng J Med 2010;363:711-23.

20. Schadendorf D, Hodi FS, Robert C, et al. Pooled analysis of long-term survival data from phase II and phase III trials of ipilimumab in unresectable or metastatic melanoma. J Clin Oncol 2015;33:1889-94.

21. Robert C, Long GV, Brady B, et al. Nivolumab in previously untreated melanoma without BRAF mutation. N Engl J Med 2015;372:320-30.

22. Motzer RJ, Escudier B, McDermott DF, et al. Nivolumab versus everolimus in advanced renal-cell carcinoma. $\mathrm{N}$ Engl J Med 2015;373:1803-13.

23. Brahmer J, Reckamp KL, Baas P, et al. Nivolumab versus docetaxel in advanced squamous-cell non-small-cell lung cancer. N Engl J Med 2015;373:123-35.

24. Borghaei H, Paz-Ares L, Horn L, et al. Nivolumab versus docetaxel in advanced non-squamous non-small-cell lung cancer. N Engl J Med 2015;373:1627-39.

25. Robert C, Schachter J, Long GV, et al. Pembrolizumab versus ipilimumab in advanced melanoma. N Engl J Med 2015;372:2521-32.

26. Zheng P, Zhou Z. Human cancer immunotherapy with PD-1/PD-L1 blockade. Biomark Cancer 2015;7:15-8.

27. Eriksson D, Stigbrand T. Radiation-induced cell death mechanisms. Tumour Biol 2010;31:363-72.

28. Kotera Y, Shimuzu K, Mule JJ. Comparative analysis of necrotic and apoptotic tumor cells as a source of antigen(s) in dendritic cell-based immunization. Cancer Res 2001;61:8105-09.

29. Garg AD, Nowis D, Golab J, et al. Immunogenic cell death, DAMPs and anticancer therapeutics: an emerging amalgamation. Biochem Biophys Acta 2010;1805:53-71.

30. Apetoh L, Ghiringhelli F, Tesniere A, et al. Toll-like receptor 4-dependent contribution of the immune system to anticancer chemotherapy and radiotherapy. Nat Med 2007;13:1050-9.

31. van der Most RG, Currie AJ, Robinson BW, et al. Decoding dangerous death: how cytotoxic chemotherapy invokes inflammation, immunity or nothing at all. Cell Death Differ 2008;15:13-20.

32. Yang Y, Liu B, Dai J, et al. Heat shock protein gp96 is a master chaperone for toll-like receptors and is important in the innate function of macrophages. Immunity 2007;26:215-26.

33. Gameiro SR, Jammeh ML, Wattenberg MM, et al. exposure, resulting in enhanced T-cell killing. Oncotarget 2014;5:403-16.

34. Ogden CA, deCathelineau A, Hoffman PR, et al. C1q and mannose binding lectin engagement of cell surface calreticulin and CD91 initiates macropinocytosis and uptake of apoptotic cells. J Exp Med 2001;194:781-95.

35. Obeid M, Panaretakis T, Joza N, et al. Calreticulin exposure is required for the immunogenicity of gammairradiation and UVC light-induced apoptosis. Cell Death Differ 2007;14:1848-50.

36. Reits EA, Hodge JW, Herberts CA, et al. Radiation modulates the peptide repertoire, enhances MHC class I expression, and induces successful antitumor immunotherapy. J Exp Med 2006;203:1259-71.

37. Desai S, Kumar A, Laskar S, et al. Cytokine profile of conditioned medium from human tumor cell lines after acute and fractionated doses of gamma radiation and its effect on survival of bystander tumor cells. Cytokine 2013;61:54-62. 
38. Matsumura S, Wang B, Kawashima N, et al. Radiationinduced CXCL16 release by breast cancer cells attracts effector T cells. J Immunol 2008;181:3099-3107.

39. Mollà M, Gironella M, Miquel R, et al. Relative roles of ICAM-1 and VCAM-1 in the pathogenesis of experimental radiation-induced intestinal inflammation. Int $\mathrm{J}$ Radiat Oncol Biol Phys 2003;57:264-73.

40. Mole RH. Whole body irradiation; radiobiology or medicine? Br J Radiol 1953;26:234-41.

41. Vatner RE, Cooper BT, Vanpouille-Box C, et al. Combinations of immunotherapy and radiation in cancer therapy. Front Oncol 2014;4:325.

42. Demaria S, Kawashima N, Yang AM, et al. Immunemediated inhibition of metastases after treatment with local radiation and CTLA-4 blockade in a mouse model of breast cancer. Clin Cancer Res 2005;11:728-34.

43. Belcaid Z, Phallen JA, Zeng J, et al. Focal radiation therapy combined with 4-1BB activation and CTLA-4 blockade yields long-term survival and a protective antigen-specific memory response in a murine glioma model. PLoS One 2014;9:e101764.

44. Verbrugge I, Hagekyriakou J, Sharp LL, et al. Radiotherapy increases the permissiveness of established mammary tumors to rejection by immunomodulatory antibodies. Cancer Res 2012;72:3163-74.

45. Zeng J, See AP, Phallen J, et al. Anti-PD-1 blockade and stereotactic radiation produce long-term survival in mice with intracranial gliomas. Int J Radiat Oncol Biol Phys 2013;86:343-9.

46. Garnett CT, Palena C, Chakraborty M, et al. Sublethal irradiation of human tumor cells modulates phenotype resulting in enhanced killing by cytotoxic $\mathrm{T}$ lymphocytes. Cancer Res 2004;64:7985-94.

47. Schaue D, Ratikan JA, Iwamoto KS, et al. Maximizing tumor immunity with fractionated radiation. Int J Radiat Oncol Biol Phys 2012;83:1306-10.

48. Lee Y, Auh SL, Wang Y, et al. Therapeutic effects of ablative radiation on local tumor require CD8+ $\mathrm{T}$ cells: changing strategies for cancer treatment. Blood 2009;114:589-95.

49. Lugade AA, Moran JP, Gerber SA, et al. Local radiation therapy of B16 melanoma tumors increases the generation of tumor antigen-specific effector cells that traffic to the tumour. J Immunol 2005;174:7516-23.

50. Dewan MZ, Galloway AE, Kawashima N, et al. Fractionated but not single-dose radiotherapy induces an immune-mediated abscopal effect when combined with anti-CTLA-4 antibody. Clin Cancer Res 2009;15:5379-88.
51. Postow MA, Callahan MK, Barker CA. Immunologic correlates of the abscopal effect in a patient with melanoma. N Engl J Med 2012;366:925-31.

52. Hiniker SM, Chen DS, Knox SJ. Abscopal effect in a patient with melanoma. N Engl J Med 2012;366:2035; author reply 2035-6.

53. Golden EB, Demaria S, Schiff PB, et al. An abscopal response to radiation and ipilimumab in a patient with metastatic non-small cell lung cancer. Cancer Immunol Res 2013;1:365-72.

54. Stamell EF, Wolchok JD, Gnjatic S, et al. The abscopal effect associated with a systemic anti-melanoma immune response. Int J Radiat Oncol Biol Phys 2013;85:293-5.

55. Silk AW, Bassetti MF, West BT, et al. Ipilimumab and radiation therapy for melanoma brain metastases. Cancer Med 2013;2:899-906.

56. Tang C, Welsh JW, de Groot P, et al. Ipilimumab with stereotactic ablative radiation therapy: phase 1 results and immunologic correlates from peripheral $\mathrm{T}$ cells. Clin Cancer Res 2017;23:1388-96.

57. Available online: https://clinicaltrials.gov/ct2/show/ NCT01497808

58. Available online: https://clinicaltrials.gov/ct2/show/ NCT01950195

59. Available online: https://clinicaltrials.gov/ct2/show/ NCT02492568

60. Available online: https://clinicaltrials.gov/ct2/show/ NCT02562625

61. Available online: https://clinicaltrials.gov/ct2/show/ NCT01703507

62. Available online: https://clinicaltrials.gov/ct2/show/ NCT01401062

63. Available online: https://clinicaltrials.gov/ct2/show/ NCT02097732

64. Available online: https://clinicaltrials.gov/ct2/show/ NCT01818986

65. Available online: https://clinicaltrials.gov/ct2/show/ NCT02298946

66. Available online: https://clinicaltrials.gov/ct2/show/ NCT01896271

67. Available online: https://clinicaltrials.gov/ct2/show/ NCT01565837

68. Available online: https://clinicaltrials.gov/ct2/show/ NCT01970527

69. Bernstein MB, Krishnan S, Hodge JW, et al. Immunotherapy and stereotactic ablative radiotherapy (iSABR): a curative approach. Nat Rev Clin Oncol 2016;13:516-24. 
70. Young K, Cottam B, Baird JR, et al. Ideal timing of immunotherapy with radiation in murine tumor models. Int J Radiat Oncol 2014;90:S58.

71. Chang JY, Liu H, Balter P, et al. Clinical outcome and predictors of survival and pneumonitis after stereotactic ablative radiotherapy for stage I non-small cell lung cancer. Radiat Oncol 2012;7:152.

72. Senthi S, Lagerwaard FJ, Haasbeek CJ, et al. Patterns of disease recurrence after stereotactic ablative radiotherapy for early stage non-small-cell lung cancer: a retrospective analysis. Lancet Oncol 2012;13:802-9.

73. Bhattacharya IS, Hoskin PJ. Stereotactic body radiotherapy for spinal and bone metastases. Clin Oncol (R Coll Radiol) 2015;27:298-306.

74. Huang F, Gang W, Yang K. Oligometastasis and oligorecurrence: more than a mirage. Radiat Oncol 2014;9:230.

75. Aitken KL, Hawkins MA. Stereotactic body radiotherapy for liver metastases. Clin Oncol (R Coll Radiol) 2015;27:307-15.

76. Tree AC, Khoo VS, Eeles RA, et al. Stereotactic body radiotherapy for oligometastases. Lancet Oncol 2013;14:e28-37.

77. Kwon ED, Drake CG, Scher HI, et al. Ipilimumab versus placebo after radiotherapy in patients with metastatic castration-resistant prostate cancer that had progressed after docetaxel chemotherapy (CA184-043): a multicentre, randomised, double-blind, phase 3 trial. Lancet Oncol 2014;15:700-12.

78. Chiocca EA, Rabkin SD. Oncolytic viruses and their application to cancer immunotherapy. Cancer Immunol

Cite this article as: Tharmalingam H, Hoskin PJ. The optimism surrounding stereotactic body radiation therapy and immunomodulation. Chin Clin Oncol 2017;6(Suppl 2):S9. doi: $10.21037 /$ cco.2017.05.01
Res 2014;2:295-300.

79. Gill S, June CH. Going viral: chimeric antigen receptor T-cell therapy for haematological malignancies. Immunol Rev 2015;263:68-89.

80. Grupp SA, Kalos M, Barrett D, et al. Chimeric antigen receptor T-cell therapy for haematological malignancies. N Engl J Med 2013;368:1509-18.

81. Guo C, Manjili MH, Subjeck JR, et al. Therapeutic cancer vaccines: past, present and future. Adv Cancer Res 2013;119:421-75.

82. Melero I, Gaudernack G, Gerritsen W, et al. Therapeutic vaccines for cancer: an overview of clinical trials. Nat Rev Clin Oncol 2014;11:509-24.

83. Small EJ, Schellhammer PF, Higano CS, et al. Placebocontrolled phase III trial of immunologic therapy with sipuleucel-T (APC8015) in patients with metastatic, asymptomatic hormone refractory prostate cancer. J Clin Oncol 2006;24:3089-94.

84. Higano CS, Schellhammer PF, Small EJ, et al. Integrated data from 2 randomized, double-blind, placebocontrolled phase 3 trials pf active cellular immunotherapy with sipuleucel-T in advanced prostate cancer. Cancer 2009;115:3670-9.

85. Kantoff PW, Higano CS, Shore ND, et al. Sipuleucel-T immunotherapy for castration-resistant prostate cancer. $\mathrm{N}$ Engl J Med 2010;363:411-22.

86. Wolchok JD, Hoos A, O’Day S, et al. Guidelines for the evaluation of immune therapy activity in solid tumors: immune-related response criteria. Clin Cancer Res 2009;15:7412-20. 